\title{
Discovery and validation of immune- related long non-coding RNA biomarkers associated with prognosis in hepatocellular carcinoma
}

Li Liu (D liuli800402@126.com )

Guizhou Medical University https://orcid.org/0000-0002-8332-8321

She Tian

Guizhou Medical University

Zhu Li

Guizhou Medical University

Yongjun Gong

Guizhou Minzu University

Hao Zhang

guizhou medical university

Primary research

Keywords: Hepatocellular carcinoma (HCC), prediction, immune-related IncRNA markers, TCGA, Molecular Signatures Database

Posted Date: March 20th, 2020

DOl: https://doi.org/10.21203/rs.3.rs-17851/v1

License: (9) (1) This work is licensed under a Creative Commons Attribution 4.0 International License. Read Full License 


\section{Abstract}

Background : Hepatocellular carcinoma (HCC) is one of the most common clinical malignant tumors, resulting in high mortality and poor prognosis. Studies have found that LncRNA plays an important role in the onset, metastasis and recurrence of hepatocellular carcinoma. The immune system plays a vital role in the development, progression, metastasis and recurrence of cancer. Therefore, immune-related IncRNA can be used as a novel biomarker to predict the prognosis of hepatocellular carcinoma.

Methods : The transcriptome data and clinical data of HCC patients were obtained by using The Cancer Genome Atlas-Liver Hepatocellular Carcinoma (TCGA-LIHC), and immune-related genes were extracted from the Molecular Signatures Database (IMMUNE RESPONSE M19817 and IMMUNE SYSTEM PROCESS M13664). By constructing the co-expression network and Cox regression analysis, 13 immune-IncRNAs was identified to predict the prognosis of HCC patients. Patients were divided into high risk group and low risk group by using the risk score formula, and the difference in overall survival (OS) between the two groups was reflected by Kaplan-Meier survival curve. The time - dependent receiver operating characteristics (ROC) analysis and principal component analysis (PCA) were used to evaluate 13 immune -IncRNAs signature.

Results : Through TCGA - LIHC extracted from 343 cases of patients with hepatocellular carcinoma RNA Seq data and clinical data, 331 immune-related genes were extracted from the Molecular Signatures Database, co-expression networks and Cox regression analysis were constructed, 13 immune-IncRNAs signature was identified as biomarkers to predict the prognosis of patients. At the same time using the risk score median divided the patients into high risk and low risk groups, and through the Kaplan-Meier survival curve analysis found that high-risk group of patients' overall survival (OS) less low risk group of patients. The AUC value of the ROC curve is 0.828 , and principal component analysis (PCA) results showed that patients could be clearly divided into two parts by immune-IncRNAs, which provided evidence for the use of 13 immune-IncRNAs signature as prognostic markers.

Conclusion : Our study identified 13 immune-IncRNAs signature that can effectively predict the prognosis of HCC patients, which may be a new prognostic indicator for predicting clinical outcomes.

\section{Background}

Hepatocellular carcinoma (HCC) is the most common histological type of primary liver cancer [1]. Worldwide, $\mathrm{HCC}$ is one of the most common malignant tumors, the fourth leading cause of cancer death, and the sixth most common malignant tumor in the world [2]. Because of its strong invasion and high incidence of metastasis, hepatocellular carcinoma often has a poor prognosis, so early diagnosis and treatment have become the key to improve the survival rate of HCC patients. It is necessary to develop efficient and non-invasive markers for early diagnosis and prognosis.

The occurrence and development of hepatocellular carcinoma (HCC) is a complex process involving multiple genes and multiple pathways [3]. Long non-coding RNA (IncRNA) is a class of regulatory 
macromolecular RNA discovered in recent years. Its transcripts were longer than 200nt. For a long time, IncRNA was considered as a meaningless transcriptional by-product [4]. Recent studies have found that IncRNA can regulate gene expression at transcriptional, post-transcriptional and epigenetic levels, and participate in many pathophysiological processes such as activation of proto-oncogenes, transcriptional activation and interference, binding and regulating the activity of corresponding proteins [5]. Current studies have shown that IncRNA plays an important role in the pathogenesis, metastasis and recurrence of hepatocellular carcinoma $[6,7]$.

The occurrence and development of hepatocellular carcinoma (HCC) is closely related to the medium stimulation in the microenvironment [8]. Hepatocellular carcinoma (HCC) is a complex ecosystem containing non-tumor cells (mainly immune-related cells), and the success of immune checkpoint suppression in solid tumors underscores the critical role of tumor microenvironment in cancer progression [9]. Therefore, immune-related IncRNAs can be used as novel biomarkers to predict the prognosis of hepatocellular carcinoma.

In this study, we through the The Cancer Genome Atlas - Liver Hepatocellular Carcinoma (TCGALIHC) and the Molecular Signatures Database to collect the transcriptome data of HCC patients clinical data and immune related genes. Through the establishment of co-expression network and Cox regression analysis, immune-related IncRNAs that can predict the prognosis of HCC patients were identified, and use the risk score to HCC patients are grouped, multivariate Cox regression analysis confirmed that the identified IncRNA has the independent prognostic role. Time-dependent receiver operating characteristics (ROC) analysis and principal component analysis (PCA) were used to further predict their prognostic ability, and gene set enrichment analysis (GSEA) was used to help explain the underlying mechanism.

\section{Materials And Methods}

\section{The patient cohort and IncRNA profiles were mined}

Transcriptome data and clinical information of Hepatocellular Carcinoma patients can be extracted from The Cancer Genome Atlas - liver Hepatocellular Carcinoma (TCGA-LIHC) (http://larssonlab.org/tcgaIncrnas/index.php) [10, 11]. After evaluating the clinical data, 343 patients with hepatocellular carcinoma (HCC) were included in the study after excluding patients who were followed up for no more than 30 days (because they were likely to die from fatal complications other than HCC) or who had RNA-seq data but no clinical prognostic information $[12,13]$. In order to identify the differential expression of IncRNA and mRNA between HCC and adjacent non-tumor tissues, the R/Bioconductor package "edgeR" was used to process the mRNA and IncRNA expression data [14]. Immune-related genes were extracted from the Molecular Signatures Database (IMMUNE RESPONSE M19817 and IMMUNE SYSTEM PROCESS M13664) [15]. Immune-related IncRNAs were identified by establishing a co-expression network. Univariate and multivariate Cox regression analysis was used to analyze the immune-related IncRNA ( $p<$ 0.05) related to the survival time of patients for model construction [16].

\section{Validation of prognostic model and survival analysis}


Immune-IncRNAs associated with prognosis were selected and the prognostic model was further constructed by multivariate Cox analysis. After the expression values of each specific gene were included, the risk score formula for each patient was constructed and weighted by its estimated regression coefficients in the multi-factor regression analysis. According to the risk score formula:

$$
\text { Risk Score (patient) }=\sum_{n=1}^{\infty} \text { coefficient }(\operatorname{lncRNAi}) \times \text { expression }(\operatorname{lncRNAi})
$$

Here, IncRNAi represents the prognostic IncRNA and expression (InRNAi) is the expression level of IncRNAi for the patient. Regression coefficient of multivariate Cox analysis was denoted as coefficient (IncRNAi) which represents the contribution of IncRNAi for prognostic risk scores [17]. Patients with higher risk scores tend to have poorer survival. HCC patients can be assigned to high-risk or low-risk groups according to the median risk as the critical point. Overall survival differences between high-risk and low-risk groups were assessed using the Kaplan-Meier method and compared using the log-rank test [18].

\section{Statistical analysis}

In order to evaluate the independence of immune-related IncRNA signature from key clinical factors, multi-factor regression analysis and stratified analysis were used to examine the role of risk score in predicting patient prognosis, hazard ratio (HR) and 95\% confidence interval (Cl) were calculated by Cox analysis, and clinical correlation analysis was performed [19]. Survival prediction comparisons based on IncRNA signature and key clinical characteristics were performed using time-dependent receiver operating characteristics (ROC) analysis [20]. Based on principal component analysis (PAC) of immune-IncRNAs and whole genes, the prognostic accuracy of the identified immune-related IncRNAs was further verified [21].

\section{Functional enrichment analysis}

Through the Gene Set Enrichment Analysis (GSEA) of Kyoto Encyclopedia of Genes and Genomes (KEGG) pathway and Gene Ontology (GO), the possible molecular mechanism of the prognosis difference between the high-risk group and the low-risk group was investigated [22, 23]. The genes were sequenced according to the degree of differential expression in the high-risk group and the low-risk group, and then the enrichment of the preset gene set was examined.

\section{Results}

The co-expression network was constructed to identify the immune-related IncRNAs signature 
Transcriptome data of 374 Hepatocellular Carcinoma (HCC) and 50 adjacent normal tissue samples were extracted from The Cancer Genome Atac-liver Hepatocellular Carcinoma Dataset (TCGA-LIHC). RNA-seq data for HCC included 14142 IncRNAs and 19,658 mRNAs expression profiles. Transcriptome data and clinical data of a total of $343 \mathrm{HCC}$ patients were included in our study according to the patients' follow-up time range of 30-3675 days and the exclusion of patients with rna-seq data but no clinical prognosis information. A total of 331 genes involved in immune-related pathways were extracted from the Molecular Signatures Database (IMMUNE RESPONSE M19817 and IMMUNE SYSTEM PROCESS M13664). Immune-related IncRNA was identified by constructing an immune co-expression network, and 542 IncRNAs were identified as immune-related (P $\leq 0.01$ ) (Table S1). The immune-related IncRNA expression was combined with the survival data, and 56 immune-related IncRNAs (Table S2) related to patient prognosis were identified by univariate Cox regression analysis and the forest map was drawn (Fig. 1). Using multi-factor Cox regression analysis, 13 immunity-related IncRNAs were further screened out for prediction model construction (Table 1), including AC080013.6, AC080013.6, AC019080.5, LINC01554, PTOV1-AS1, MIR210HG, MSC-AS1, AL049840.2, AC023157.3, AL031985.3, AC145207.5, AC010761.1, and Z83851.2.

\section{Prognostic validation of 13 immune-related IncRNAs signature in HCC patients}

In order to establish the focused expression characteristics of immune-related IncRNAs for survival prediction, the expression profiles of 13 immune-Incrnas signatures with independent prognosis were selected to establish a multivariate Cox regression model to evaluate their relative predictive power. In the above multi-factor Cox analysis, the expression level of immune-related IncRNAs with independent prognosis and the weighted method of regression coefficient were used to characterize 13 immunerelated IncRNAs signature. The estimation method of prognostic risk score was as follows: Risk score (patient) $=(0.4156 \times$ expression value of AC080013.6区 $+\otimes 0.1793 \times$ expression value of PRRT3-

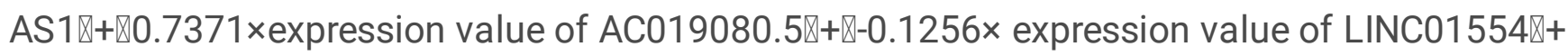

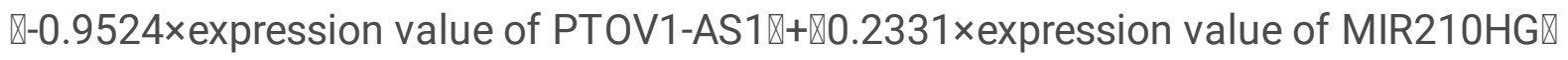
$+\llbracket 0.4169 \times$ expression value of MSC-AS1 $₫+\rrbracket 0.8936 \times$ expression value of

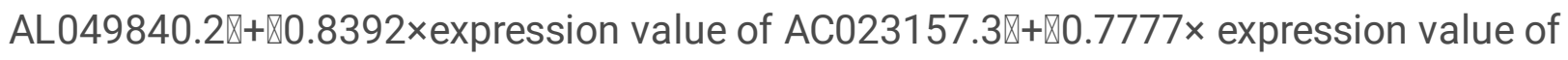

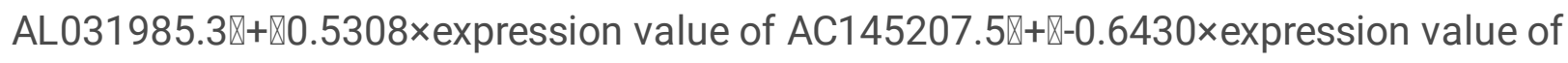
AC010761.1凶+ष0.5159xexpression value of Z83851.2邓. Calculate each of the prognosis of patients with risk score, according to the score of the median value, 343 patients were divided into high-risk and lowrisk group (Fig. 2A), at the same time to calculate the risk score is associated with cancer death (Fig. 2B), the relationship between as shown in the heat map (Fig. 2C), LINC01554 expression level increased with the increase of risk score gradually reduce, the rest of the immune related IncRNA expression level gradually increased with the increase of the risk score. Kaplan-meier analysis was used to evaluate the difference in survival between the high-risk group and the low-risk group (Fig. 2D), and the results showed that the overall survival (OS) of the high-risk group was worse than that of the low-risk group.

\section{Evaluation of 13 immune-related IncRNAs signature models}


Univariate and multivariate Cox regression methods were used to determine whether 13 immune-related IncRNAs signature could be used as independent predictors of HCC patients. According to the results of univariate analysis, risk score was significantly correlated with prognosis. After multivariate adjustment using the above factors, the risk score remained a reliable and independent predictor of risk in the cohort $(P<0.001)$ (Table 2). Based on univariate and multivariate analyses, we constructed a forest graph (Fig. $3 \mathrm{~A}, \mathrm{~B}$ ) that included independent prognostic factors (risk score, age, gender, grade, stage and TMN staging). The prognostic effects of 13 immune-related IncRNAs signature was evaluated by timedependent receiver operating characteristics (ROC) analysis and calculating the area under the ROC curve (AUC) (Fig.3C). The results showed that the AUC of 13 immune-related IncRNA signature was 0.828, showing higher specificity and sensitivity compared with other independent prognostic factors. At the same time, the clinical correlation between 13 immune-IncRNAs signature and T staging was analyzed (Fig.3D), and it was found that AC010761.1, AC023157.3, AC145207.5, AL031985.3, LINC01554, MIR210HG, and ptov1-as1 were correlated with T staging. Through principal component analysis (PCA), based on the 13 immune related IncRNAs signature (Fig. 4A) and the gene expression profile (Fig. 4B) studies the difference between low risk and high risk groups, show that low risk and high risk groups are usually distributed in different directions, the risk gene can significantly HCC patients can be divided into two parts, also for the identification of 13 immune related IncRNAs signature as to provide evidence for the HCC patients prognosis.

\section{Possible molecular mechanisms underlying the differential prognosis of HCC patients}

Further executive function was performed by gene set enrichment analysis (GSEA) (select TOP6 for display), Gene Set Enrichment Analysis (GSEA) of Kyoto Encyclopedia of Genes and Genomes (KEGG) pathway indicated significant enrichment of immune phenotype $\triangle T a b l e 3 \rrbracket F i g .5 \rrbracket$ : Progesterone mesiated oocyte maturation, Ubiquitin mediated proteolysis, Oocyte meiosis, Cell cycle, Inositol phosphate metabolism, Vasopressin regulated water reabsorption. Gene Set Enrichment Analysis (GSEA) of Gene Ontology (GO) indicated significant enrichment of immune phenotype区Table 4囚Fig. 6囚: Spliceosomal complex assembly, Chromatin remodeling, Peptidyl lysine modification, Regulation of generation of precursor metabolites and energy, Import into nucleus, Positive regulation of cell cycle phase transition. These findings provide evidence for further exploration of the possible molecular mechanisms underlying the prognostic differences in HCC patients.

\section{Discussion}

Hepatocellular carcinoma (HCC) is one of the most common malignant tumors in clinical practice and the third leading cause of cancer death, accounting for more than $80 \%$ of the incidence of liver cancer [24]. Hepatocellular carcinoma is characterized by strong invasiveness and high incidence of metastasis, resulting in high mortality and poor prognosis, with a 5 -year survival rate of only $10 \%-30 \%[25,26]$. Early diagnosis and treatment have become the key to improve the survival rate of HCC patients. At present, common clinical markers of liver cancer include alpha fetoprotein(AFP) $\square$ desrcarboxy prothrombin $\square$ DCP) $\square$ squamous cell carcinoma antigen-IgM complexes $\square$ SCCA-IgMCs), etc $[27,28]$. Although these markers 
have some specificity in the diagnosis of HCC, they are less sensitive for early diagnosis, and HCC is usually diagnosed at an advanced stage. Therefore, it is necessary to develop efficient and non-invasive new markers for early diagnosis and prognosis.

Tumor microenvironment (TME) is a complex integrated system of tumor cell growth $[29,30]$. This microenvironment is composed of tumor constituent cells, endothelial cells, immune cells, fibroblasts and extracellular matrix [31]. The immune system plays an important role in the tumor microenvironment and plays a vital role in the development, progression, metastasis and recurrence of cancer [32]. Cancer cells and immune cells show metabolic reprogramming in the tumor microenvironment, which is closely related to immune cell function and edits tumor immunology [33]. The latest findings in immune cell metabolism offer broad prospects for clinical therapies to treat cancer and will be critical to the development of identifying biomarkers $[9,34]$.

LncRNAs are a class of RNAs that are longer than 200 nucleotides but do not encode proteins [35]. Studies have proved that its expression is tissue-specific, plays an important role in the life process, and participates in almost all aspects of cell life activities. It can participate in the regulation of cell proliferation, cell differentiation and other life activities at epigenetic level, transcriptional level and posttranscriptional level $[36,37]$. Therefore, abnormal expression of IncRNA is closely related to the occurrence and development of many human diseases, including tumors [38, 39]. Many studies have proved that IncRNAs can regulate gene expression through interaction with proteins or RNA, thus regulating cell proliferation, apoptosis and metastasis and participating in the occurrence and development of HCC [40]. Differential expression of IncRNAs in tissues can distinguish between cancer tissues and adjacent tissues, indicating that IncRNAs in cancer tissues have a characteristic expression profile [41]. At present, the abnormal expression of many IncRNAs in hepatocellular carcinoma has been identified. In HCC tumor tissues, the up-regulation of IncRNAs such as H19 and HOTAIR is associated with distant metastasis, and the down-regulation of IncRNA such as MEG3 is associated with cell proliferation [42-44]. Since the abnormal expression of some IncRNAs has been proved to be closely related to cancer, the use of IncRNAs as a marker for cancer diagnosis and prognosis has also become a new research direction [45]. Currently, immune-related IncRNA has not been reported as a prognostic marker for HCC patients.

In this study, we obtained transcriptome information and clinical data of a HCC patient using TCGA-LIHC Database, and obtained immune-related genes through Molecular Signatures Database. Through the establishment of co-expression network and Cox regression analysis, 13 immune-related IncRNAs signature was identified to predict the prognosis of HCC patients. Risk score was used to distinguish HCC patients into high-risk group and low-risk group, and the differential expression of 13 immune-IncRNAs signature in the two groups was analyzed. Kaplan-Meier survival chart was used to analyze the difference in survival status between high-risk group and low-risk group. Using univariate and multivariate Cox regression analysis, to determine the 13 kinds of immune related IncRNA can serve as an independent predictor of $\mathrm{HCC}$ of patients, and use the time-dependent receiver operating characteristics (ROC) curve, AUC value of 0.828 , to evaluate the immune - IncRNAs on overall survival of predictive value, through the 
principal component analysis (PCA) has once again proven its predictive value. Gene set enrichment analysis (GSEA) was used to investigate the possible molecular mechanism of the difference in prognosis between the high-risk group and the low-risk group.

\section{Conclusions}

In summary, we identified 13 immune-related IncRNAs signature by constructing a co-expression network that can effectively predict the prognosis of HCC patients, which may become a new prognostic indicator for predicting clinical outcomes and contribute to the clinical decision-making of individual treatment. It is worth further study on the molecular mechanism of hepatocellular carcinoma.

\section{Declarations}

\section{Ethics approval and consent to participate}

This study was reviewed and approved by the Medical Ethics Committee of Renmin hospital of Guizhou medical university, Guiyang, China.

\section{Consent for Publication}

All authors have agreed to publish this manuscript.

\section{Availability of data and materials}

All data generated or analyzed during this study are included in this published article.

\section{Competing interests}

The authors declare that they have no conflicts of interests.

\section{Funding}

This study was supported by the "Xin Miao" Foundation of Guizhou Medical University, NO:5779-10

\section{Authors' contributions}


Li Liu conceived and designed the experiments. Hao Zhang performed the experiments. She Tian and Zhu Li contributed reagents/materials/analysis tools. Li Liu wrote the paper. All authors read and approved the final manuscript.

\section{Acknowledgements}

Not applicable.

\section{References}

1. Grandhi MS, Kim AK, Ronnekleiv-Kelly SM, Kamel IR, Ghasebeh MA, Pawlik TM: Hepatocellular carcinoma: From diagnosis to treatment. Surg Oncol 2016, 25(2):74-85.

2. Villanueva A: Hepatocellular Carcinoma. N Engl J Med 2019, 380(15):1450-1462.

3. Thurnherr T, Mah WC, Lei Z, Jin Y, Rozen SG, Lee CG: Differentially Expressed miRNAs in Hepatocellular Carcinoma Target Genes in the Genetic Information Processing and Metabolism Pathways. Sci Rep 2016, 6:20065.

4. Quinn JJ, Chang HY: Unique features of long non-coding RNA biogenesis and function. Nat Rev Genet 2016, 17(1):47-62.

5. Sanchez Calle A, Kawamura Y, Yamamoto Y, Takeshita F, Ochiya T: Emerging roles of long noncoding RNA in cancer. Cancer Sci 2018, 109(7):2093-2100.

6. Liu X, Liang Y, Song R, Yang G, Han J, Lan Y, Pan S, Zhu M, Liu Y, Wang Y et al: Long non-coding RNA NEAT1-modulated abnormal lipolysis via ATGL drives hepatocellular carcinoma proliferation. $\mathrm{Mol}$ Cancer 2018, 17(1):90.

7. Wang Y, Liu Z, Yao B, Li Q, Wang L, Wang C, Dou C, Xu M, Liu Q, Tu K: Long non-coding RNA CASC2 suppresses epithelial-mesenchymal transition of hepatocellular carcinoma cells through CASC2/miR-367/FBXW7 axis. Mol Cancer 2017, 16(1):123.

8. Novikova MV, Khromova NV, Kopnin PB: Components of the Hepatocellular Carcinoma Microenvironment and Their Role in Tumor Progression. Biochemistry (Mosc) 2017, 82(8):861-873.

9. Nishida N, Kudo M: Immunological Microenvironment of Hepatocellular Carcinoma and Its Clinical Implication. Oncology 2017, 92 Suppl 1:40-49.

10. Tomczak K, Czerwinska P, Wiznerowicz M: The Cancer Genome Atlas (TCGA): an immeasurable source of knowledge. Contemp Oncol (Pozn) 2015, 19(1A):A68-77.

11. Blum A, Wang P, Zenklusen JC: SnapShot: TCGA-Analyzed Tumors. Cell 2018, 173(2):530.

12. He Y, Dang Q, Li J, Zhang Q, Yu X, Xue M, Guo W: Prediction of hepatocellular carcinoma prognosis based on expression of an immune-related gene set. Aging (Albany NY) 2020, 12(1):965-977.

13. Wang Y, Xu M, Yang Q: A six-microRNA signature predicts survival of patients with uterine corpus endometrial carcinoma. Curr Prob/ Cancer 2019, 43(2):167-176. 
14. Ouyang D, Li R, Li Y, Zhu X: A 7-IncRNA signature predict prognosis of Uterine corpus endometrial carcinoma. J Cell Biochem 2019, 120(10):18465-18477.

15. Wang W, Zhao Z, Yang F, Wang H, Wu F, Liang T, Yan X, Li J, Lan Q, Wang J et al: An immune-related IncRNA signature for patients with anaplastic gliomas. J Neurooncol 2018, 136(2):263-271.

16. Wu Y, Wang PS, Wang BG, Xu L, Fang WX, Che XF, Qu XJ, Liu YP, Li Z: Genomewide identification of a novel six-LncRNA signature to improve prognosis prediction in resectable hepatocellular carcinoma. Cancer Med 2018, 7(12):6219-6233.

17. Zhou M, Zhang Z, Zhao H, Bao S, Sun J: A novel IncRNA-focus expression signature for survival prediction in endometrial carcinoma. BMC Cancer 2018, 18(1):39.

18. Lin T, Fu Y, Zhang X, Gu J, Ma X, Miao R, Xiang X, Niu W, Qu K, Liu C et al: A seven-long noncoding RNA signature predicts overall survival for patients with early stage non-small cell lung cancer. Aging (Albany NY) 2018, 10(9):2356-2366.

19. Zhu Q, Yang H, Cheng P, Han Q: Bioinformatic analysis of the prognostic value of the IncRNAs encoding snoRNAs in hepatocellular carcinoma. Biofactors 2019, 45(2):244-252.

20. Zhao QJ, Zhang J, Xu L, Liu FF: Identification of a five-long non-coding RNA signature to improve the prognosis prediction for patients with hepatocellular carcinoma. World J Gastroentero/ 2018, 24(30):3426-3439.

21. Miller JP, Ramaswamy R, Akinwande O: Using principal component analysis for the prediction of tumor response to transarterial chemoembolization. Abdom Radiol (NY) 2019, 44(7):2594-2601.

22. Subramanian A, Tamayo P, Mootha VK, Mukherjee S, Ebert BL, Gillette MA, Paulovich A, Pomeroy SL, Golub TR, Lander ES et al: Gene set enrichment analysis: a knowledge-based approach for interpreting genome-wide expression profiles. Proc Natl Acad Sci U S A 2005, 102(43):15545-15550.

23. Mootha VK, Lindgren CM, Eriksson KF, Subramanian A, Sihag S, Lehar J, Puigserver P, Carlsson E, Ridderstrale M, Laurila E et al: PGC-1alpha-responsive genes involved in oxidative phosphorylation are coordinately downregulated in human diabetes. Nat Genet 2003, 34(3):267-273.

24. Ji J, Chen H, Liu XP, Wang YH, Luo CL, Zhang WW, Xie W, Wang FB: A miRNA Combination as Promising Biomarker for Hepatocellular Carcinoma Diagnosis: A Study Based on Bioinformatics Analysis. J Cancer 2018, 9(19):3435-3446.

25. Ha J, Yan M, Aguilar M, Bhuket T, Tana MM, Liu B, Gish RG, Wong RJ: Race/ethnicity-specific disparities in cancer incidence, burden of disease, and overall survival among patients with hepatocellular carcinoma in the United States. Cancer 2016, 122(16):2512-2523.

26. Zeng H, Chen W, Zheng R, Zhang S, Ji JS, Zou X, Xia C, Sun K, Yang Z, Li H et al: Changing cancer survival in China during 2003-15: a pooled analysis of 17 population-based cancer registries. Lancet Glob Health 2018, 6(5):e555-e567.

27. Hung $\mathrm{CH}$, Hu TH, Lu SN, Kuo FY, Chen CH, Wang JH, Huang CM, Lee CM, Lin CY, Yen YH et al: Circulating microRNAs as biomarkers for diagnosis of early hepatocellular carcinoma associated with hepatitis B virus. Int J Cancer 2016, 138(3):714-720. 
28. Zhang Z, Zhang Y, Wang Y, Xu L, Xu W: Alpha-fetoprotein-L3 and Golgi protein 73 may serve as candidate biomarkers for diagnosing alpha-fetoprotein-negative hepatocellular carcinoma. Onco Targets Ther 2016, 9:123-129.

29. Wu T, Dai Y: Tumor microenvironment and therapeutic response. Cancer Lett 2017, 387:61-68.

30. Meurette O, Mehlen P: Notch Signaling in the Tumor Microenvironment. Cancer Cel/ 2018, 34(4):536548.

31. Tahmasebi Birgani M, Carloni V: Tumor Microenvironment, a Paradigm in Hepatocellular Carcinoma Progression and Therapy. Int J Mol Sci 2017, 18(2).

32. Kurebayashi Y, Ojima H, Tsujikawa H, Kubota N, Maehara J, Abe Y, Kitago M, Shinoda M, Kitagawa Y, Sakamoto M: Landscape of immune microenvironment in hepatocellular carcinoma and its additional impact on histological and molecular classification. Hepatology 2018, 68(3):1025-1041.

33. Dong LQ, Peng LH, Ma LJ, Liu DB, Zhang S, Luo SZ, Rao JH, Zhu HW, Yang SX, Xi SJ et al: Heterogeneous immunogenomic features and distinct escape mechanisms in multifocal hepatocellular carcinoma. J Hepatol 2019.

34. Sia D, Jiao Y, Martinez-Quetglas I, Kuchuk O, Villacorta-Martin C, Castro de Moura M, Putra J, Camprecios $\mathrm{G}$, Bassaganyas L, Akers $\mathrm{N}$ et al: Identification of an Immune-specific Class of Hepatocellular Carcinoma, Based on Molecular Features. Gastroenterology 2017, 153(3):812-826.

35. Wang Y, Yang L, Chen T, Liu X, Guo Y, Zhu Q, Tong X, Yang W, Xu Q, Huang D et al: A novel IncRNA MCM3AP-AS1 promotes the growth of hepatocellular carcinoma by targeting miR-194-5p/FOXA1 axis. Mol Cancer 2019, 18(1):28.

36. Ponting CP, Oliver PL, Reik W: Evolution and functions of long noncoding RNAs. Cel/ 2009, 136(4):629-641.

37. Fatica A, Bozzoni I: Long non-coding RNAs: new players in cell differentiation and development. Nat Rev Genet 2014, 15(1):7-21.

38. Huarte M: The emerging role of IncRNAs in cancer. Nat Med 2015, 21(11):1253-1261.

39. Schmitt AM, Chang HY: Long Noncoding RNAs in Cancer Pathways. Cancer Cell 2016, 29(4):452463.

40. Huang JL, Zheng L, Hu YW, Wang Q: Characteristics of long non-coding RNA and its relation to hepatocellular carcinoma. Carcinogenesis 2014, 35(3):507-514.

41. Kamel MM, Matboli M, Sallam M, Montasser IF, Saad AS, El-Tawdi AHF: Investigation of long noncoding RNAs expression profile as potential serum biomarkers in patients with hepatocellular carcinoma. Trans/ Res 2016, 168:134-145.

42. Ye Y, Guo J, Xiao P, Ning J, Zhang R, Liu P, Yu W, Xu L, Zhao Y, Yu J: Macrophages-induced long noncoding RNA H19 up-regulation triggers and activates the miR-193b/MAPK1 axis and promotes cell aggressiveness in hepatocellular carcinoma. Cancer Lett 2020, 469:310-322.

43. Yang T, He X, Chen A, Tan K, Du X: LncRNA HOTAIR contributes to the malignancy of hepatocellular carcinoma by enhancing epithelial-mesenchymal transition via sponging miR-23b-3p from ZEB1. 
Gene 2018, 670:114-122.

44. Zhang Y, Liu J, Lv Y, Zhang C, Guo S: LncRNA meg3 suppresses hepatocellular carcinoma in vitro and vivo studies. Am J Trans/ Res 2019, 11(7):4089-4099.

45. Dragomir MP, Kopetz S, Ajani JA, Calin GA: Non-coding RNAs in GI cancers: from cancer hallmarks to clinical utility. Gut 2020.

\section{Tables}

Due to technical limitations, the tables are only available as a download in the supplemental files section.

Table1: Using multivariate Cox regression analysis, 13 immune-related IncRNAs signature was finally identified to predict the prognosis of HCC patients.

Table2: A list of independent prognostic factors associated with HCC patients identified by univariate and multivariate Cox regression analysis.

Table 3: GSEA-KEGG analysis checklist.

Table 4: GSEA-GO analysis checklist.

\section{Supplemental Tables}

TableS1囚A list of 542 immune-related IncRNAs was identified in hepatocellular carcinoma by constructing a co-expression network.

Tables2 $\triangle$ A list of 56 immune-Incrnas associated with the prognosis of HCC patients was identified by univariate Cox regression analysis.

\section{Figures}




\begin{tabular}{|c|c|c|}
\hline $\begin{array}{l}06815 \\
02640\end{array}$ & 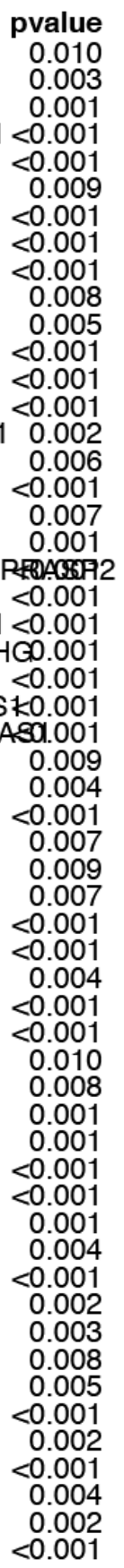 & $\begin{array}{l}\text { Hazard ratio } \\
567(1.114-2.205) \\
746(0.615-0.904) \\
485(1.165-1.893) \\
435(1.187-1.736) \\
886(1.329-2.676) \\
759(1.148-2.694) \\
556(1.214-1.994) \\
136(1.505-3.031) \\
480(1.493-4.121) \\
600(1.130-2.265) \\
378(1.100-1.726) \\
528(1.263-1.849) \\
934(1.337-2.798) \\
454(0.293-0.706) \\
672(1.201-2.329) \\
891(0.819-0.968) \\
514(1.199-1.913) \\
205(1.052-1.379) \\
060(1.327-3.198) \\
937(1.569-5.497) \\
156(1.608-2.890) \\
611(1.277-2.032) \\
501(1.175-1.917) \\
697(1.349-2.134) \\
049(1.385-3.033) \\
930(1.329-2.803) \\
721(1.146-2.584) \\
452(1.126-1.874) \\
323(1.449-3.724) \\
405(1.098-1.799) \\
732(0.580-0.924) \\
164(1.238-3.782) \\
553(1.251-1.929) \\
584(1.301-1.928) \\
610(1.164-2.227) \\
725(1.383-2.152) \\
598(2.109-6.138) \\
327(1.070-1.645) \\
750(1.159-2.642) \\
857(1.269-2.717) \\
341(1.389-3.943) \\
914(1.376-2.663) \\
471(2.347-5.133) \\
901(1.282-2.819) \\
406(1.116-1.770) \\
534(1.654-3.881) \\
303(1.102-1.541) \\
721(1.207-2.453) \\
415(1.093-1.832) \\
516(1.137-2.022) \\
844(1.553-5.208) \\
107(1.311-3.385) \\
777(1.255-2.407) \\
121(1.199-2.634) \\
659(0.535-3.413)\end{array}$ \\
\hline
\end{tabular}

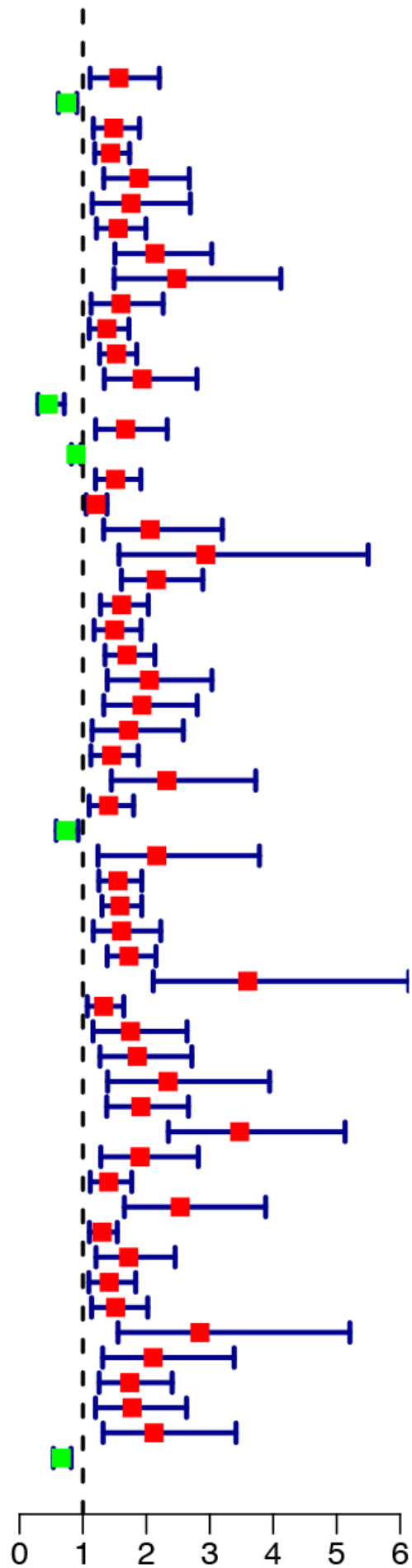

Hazard ratio

\section{Figure 1}

A forest map of 56 prognostic related immune-IncRNAs. 

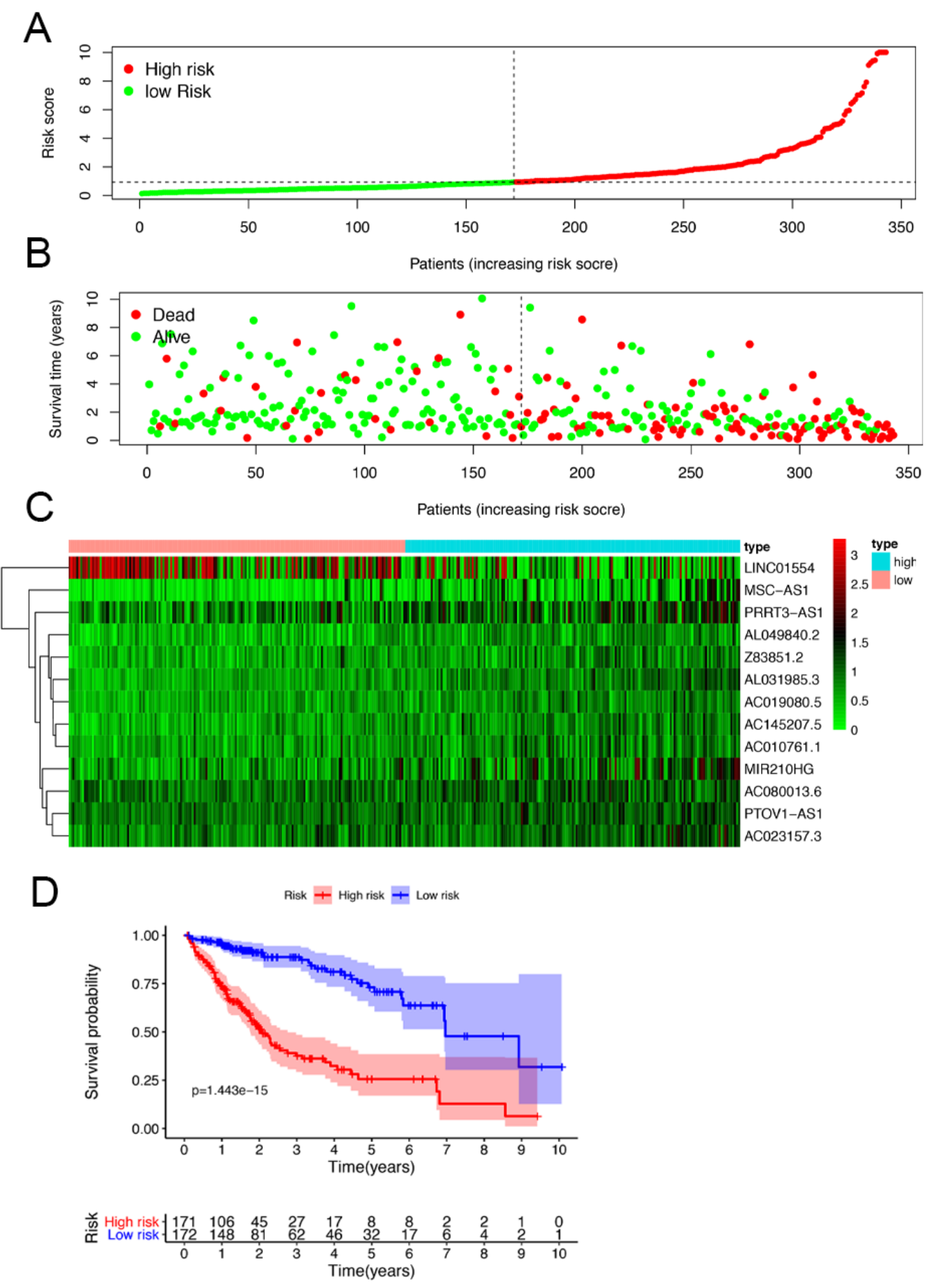

\section{Figure 2}

Risk score analysis of 13 immune-IncRNAs signature. (A) HCC patients were divided into two groups according to the median value (high risk group and low risk group). (B) The relationship between risk scores and cancer-related deaths. (C) heat maps of the expression of 13 immune-related IncRNAs identified in the high-risk and low-risk groups. (D) Kaplan-Meier analysis was used to compare the overall 
survival (OS) of the high-risk group of HCC patients with the low-risk group, and the overall survival (OS) of the high-risk group was worse.

A

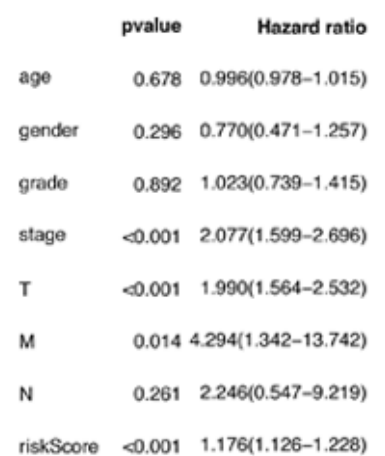

riskScore $<0.001 \quad 1.176(1.126-1.228)$

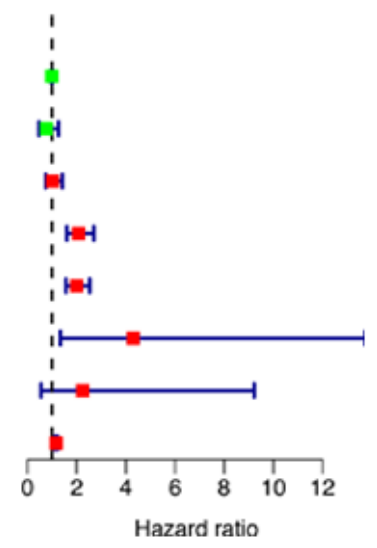

C

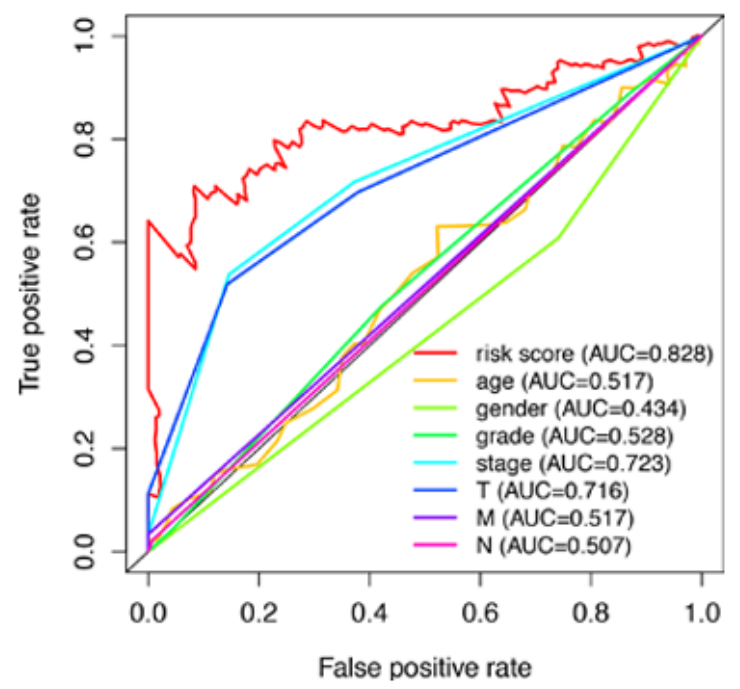

B

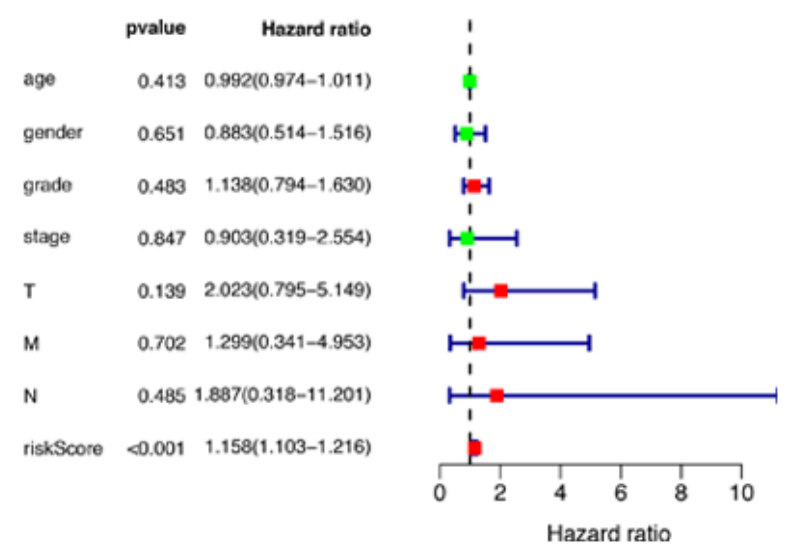

D

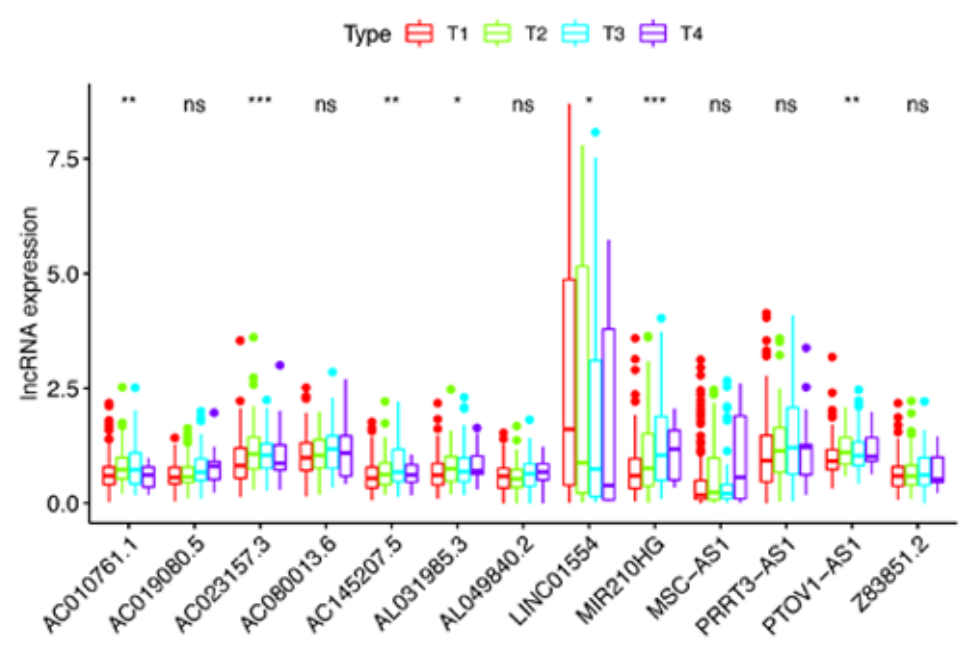

\section{Figure 3}

Forest map of independent prognostic factors $囚$ the time-dependent receiver operating characteristics (ROC) curve. (A, B) Univariate and multivariate Cox regression analysis was used to identify independent predictors of HCC patients, and $p<0.001$ was statistically significant. (C) The time-dependent receiver operating characteristics (ROC) curve. (D) Expression of 13 immune-related IncRNAs in T staging. ${ }^{\star} P<0.05,{ }^{*} \mathrm{P}<0.01, * \star * P<0.001, * \star \star * P<0.0001$. 
A

- Low risk • High risk

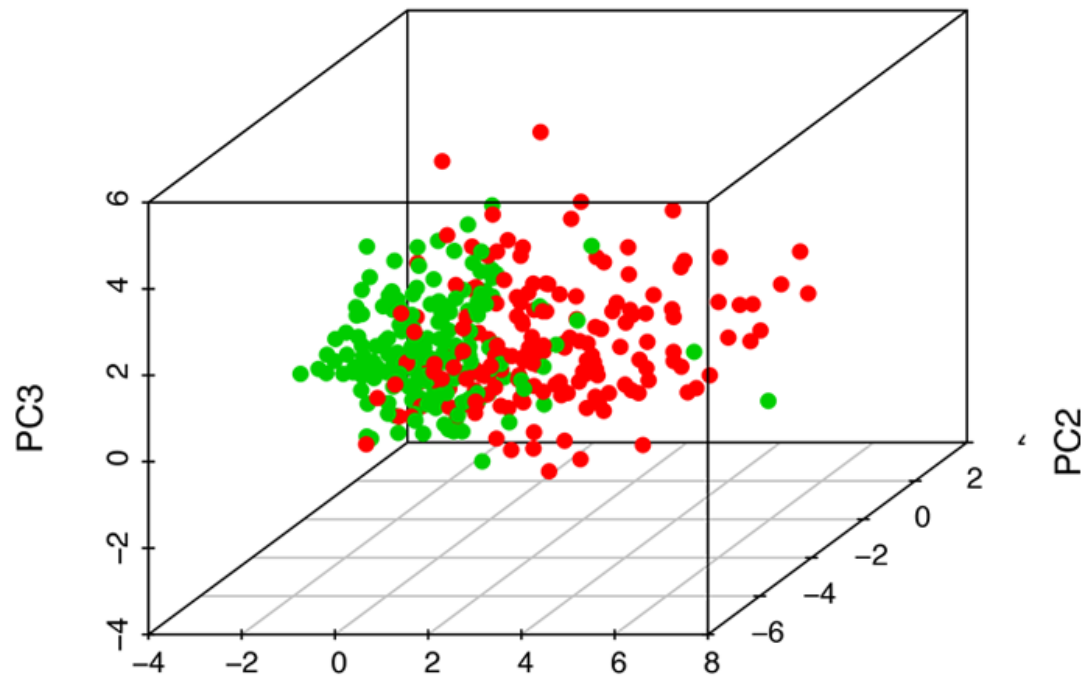

PC1

B

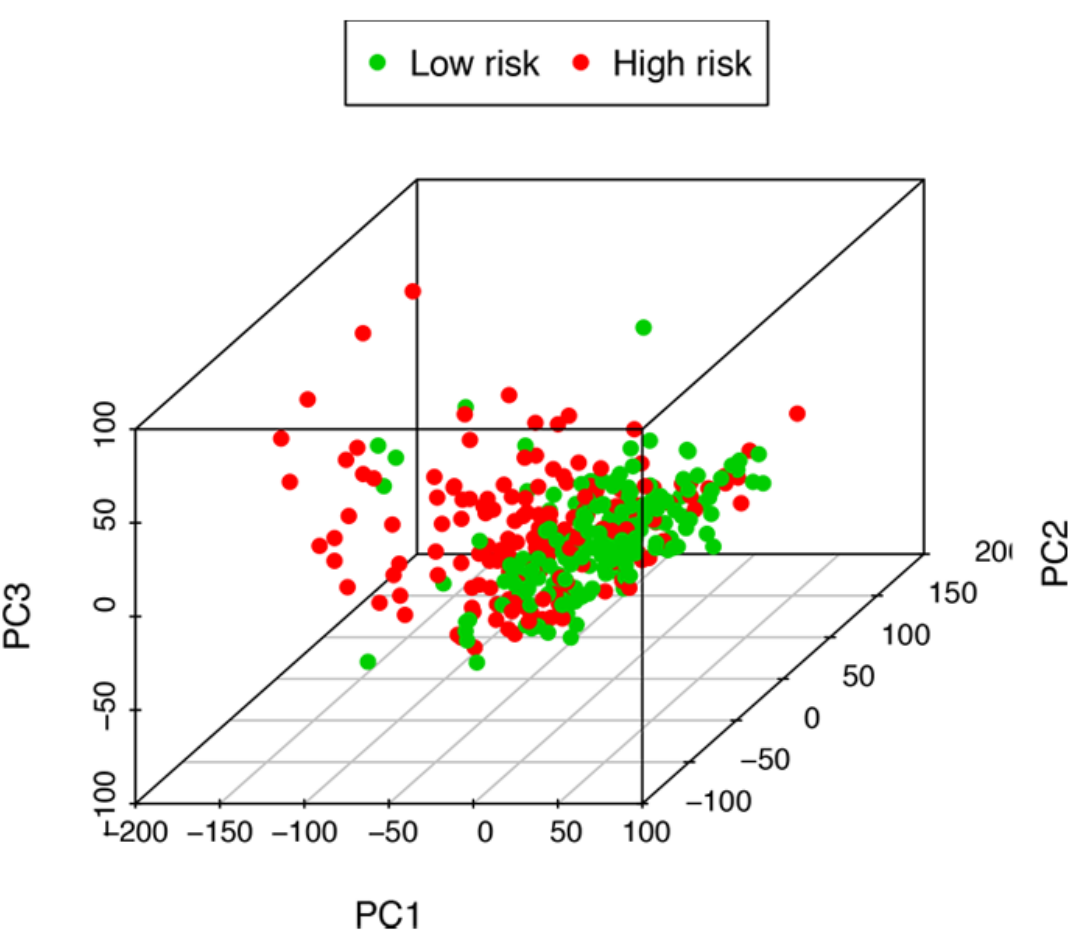

Figure 4

Principal component analysis (PCA). (A) Principal component analysis based on 13 immune-related IncRNAs signature. (B) Principal component analysis based on whole gene expression profile. 
A

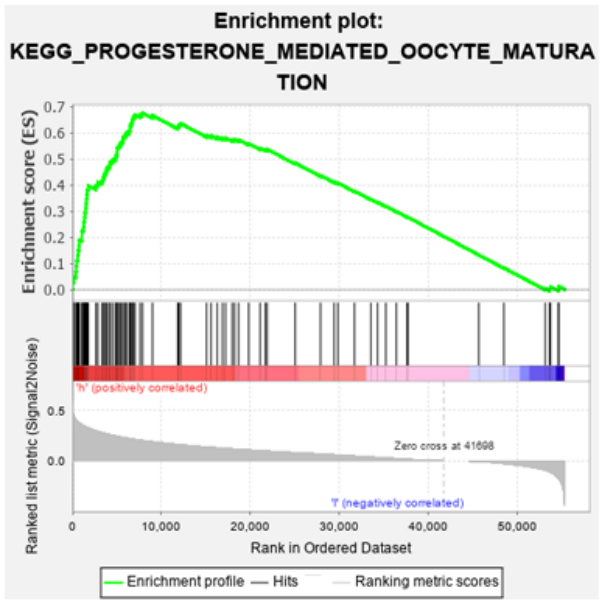

C

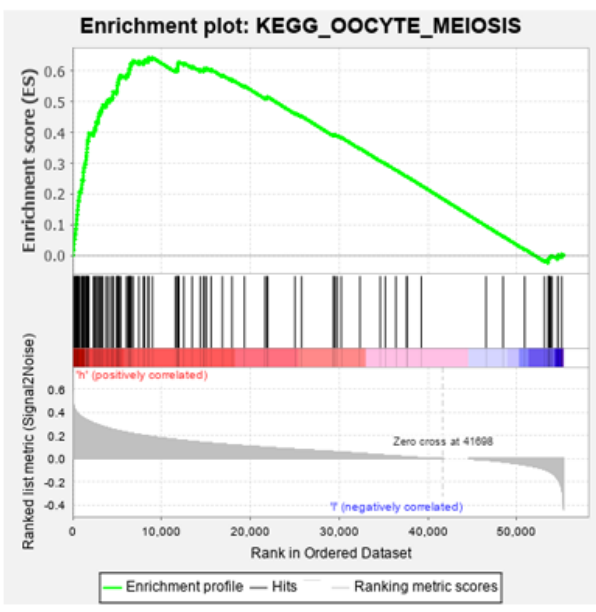

$E$

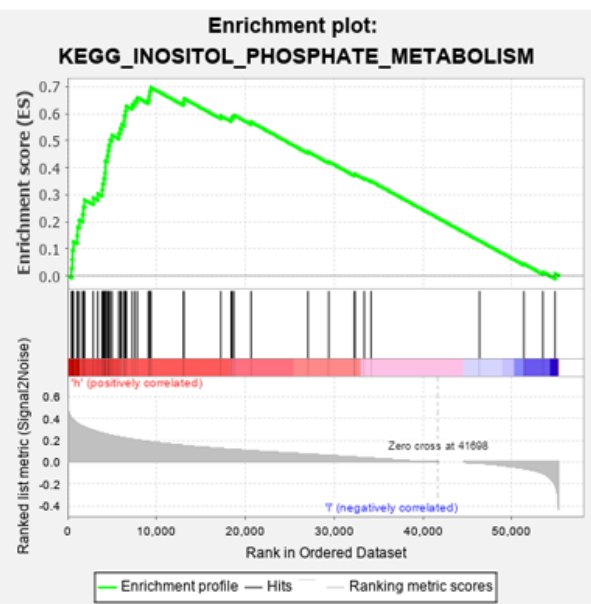

B

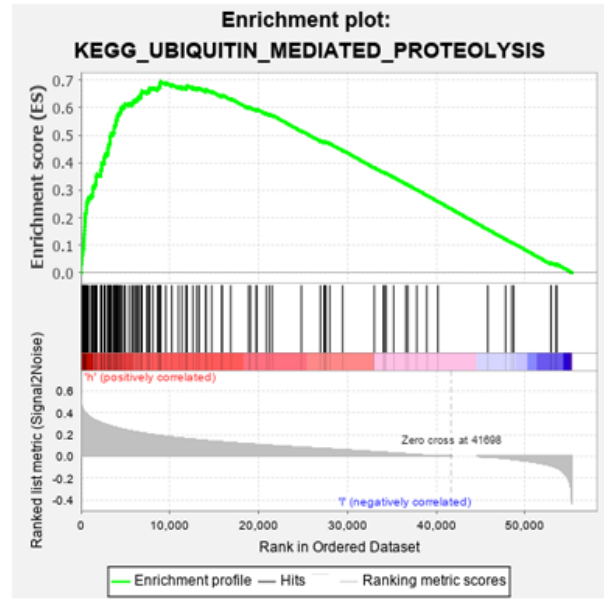

D

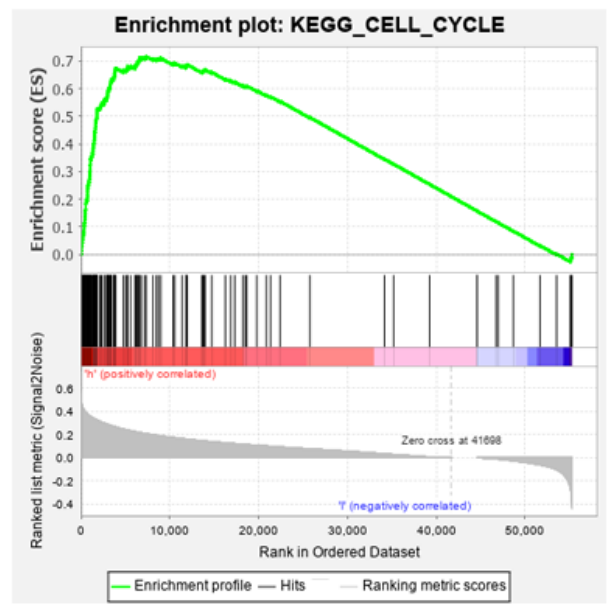

$\mathrm{F}$

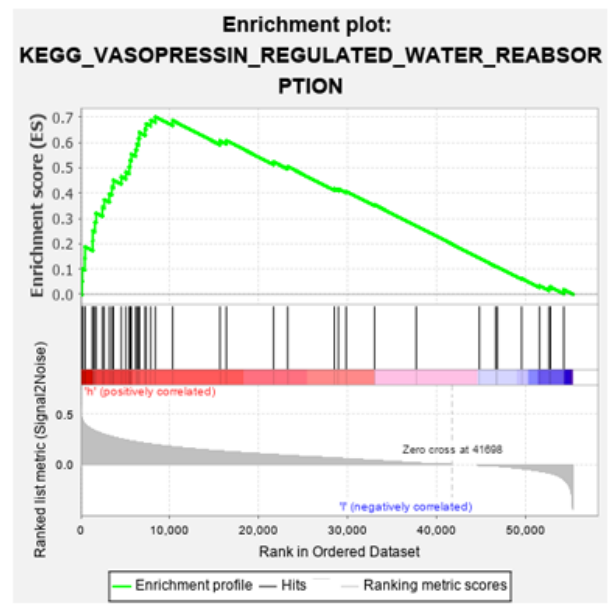

\section{Figure 5}

Gene Set Enrichment Analysis (GSEA) of Kyoto Encyclopedia of Genes and Genomes (KEGG) pathway indicated significant enrichment of immune phenotype. (A) Progesterone mesiated oocyte maturation, (B) Ubiquitin mediated proteolysis, (C) Oocyte meiosis, (D) Cell cycle, (E) Inositol phosphate metabolism, (F) Vasopressin regulated water reabsorption. 
A

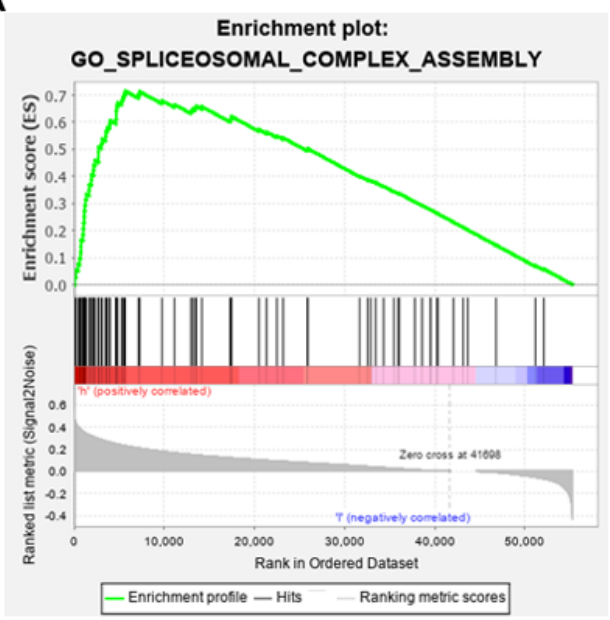

C

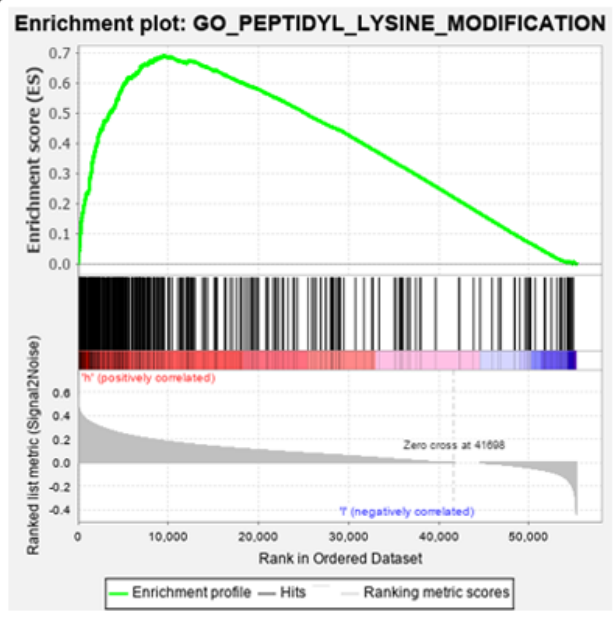

$E$

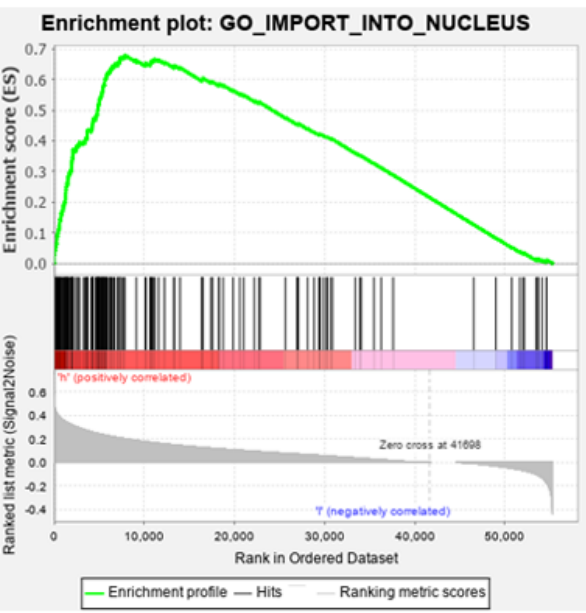

$\mathrm{B}$

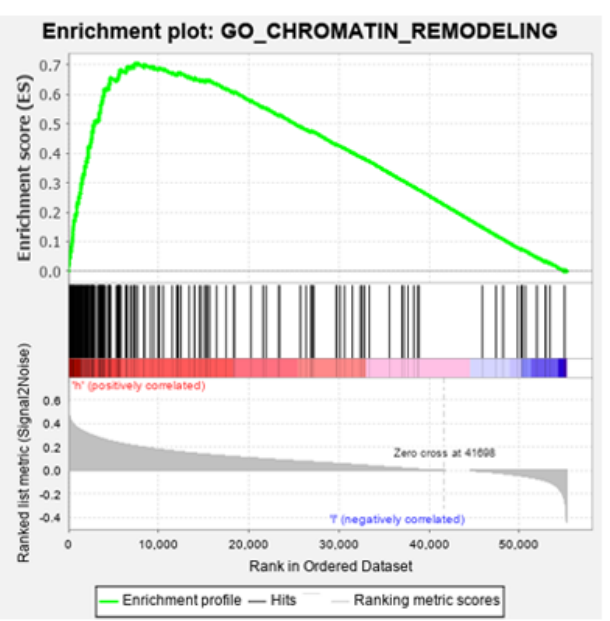

D Enrichment plot:
GO_REGULATION_OF_GENERATION_OF_PRECURSOR METABOLITES_AND_ENERGY

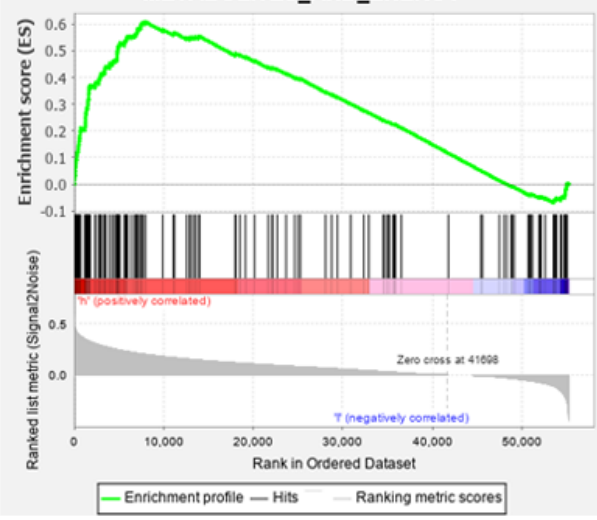

$\mathrm{F}$

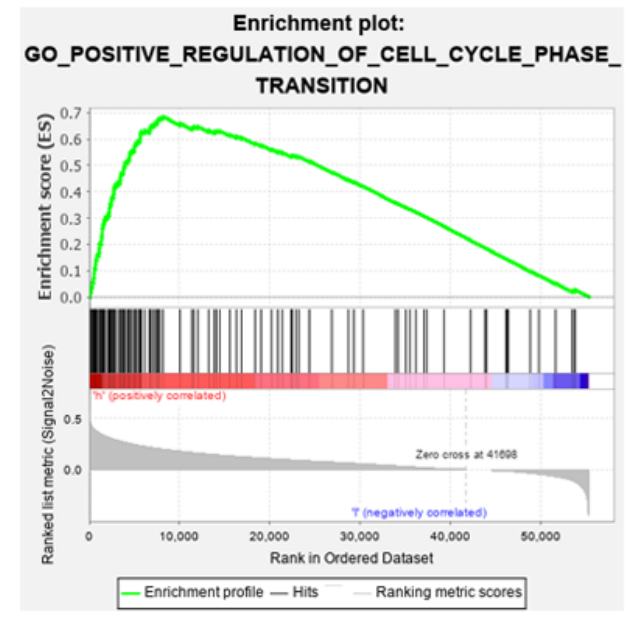

\section{Figure 6}

Gene Set Enrichment Analysis (GSEA) of Gene Ontology (GO) indicated significant enrichment of immune phenotype. (A) Spliceosomal complex assembly, (B) Chromatin remodeling, (C) Peptidyl lysine modification, (D) Regulation of generation of precursor metabolites and energy, (E) Import into nucleus, (F) Positive regulation of cell cycle phase transition. 


\section{Supplementary Files}

This is a list of supplementary files associated with this preprint. Click to download.

- Table.S1.xlsx

- Table3.xlsx

- Table4.xlsx

- TableS2.xIsx

- Table1.xlsx

- Table2.xlsx 